\title{
The SGLT2 inhibitor dapagliflozin attenuates the activity of ROS-NLRP3 inflammasome axis in steatohepatitis with diabetes mellitus
}

\author{
Weiling Leng ${ }^{1 \#}$, Mingxia Wu ${ }^{2 \#}$, Hang Pan ${ }^{1 \#}$, Xiaotian Lei ${ }^{1}$, Liu Chen ${ }^{1}$, Qinan $W^{3}$, Xinshou Ouyang ${ }^{4}$, \\ Ziwen Liang ${ }^{1}$
}

${ }^{1}$ Department of Endocrinology, ${ }^{2}$ Health Management Center, Southwest Hospital, the Third Military Medical University (Army Medical University), Chongqing 400038, China; ${ }^{3}$ Department of Endocrine Nephropathy, Chongqing University Cancer Hospital and Chongqing Cancer Institute and Chongqing Cancer Hospital, Chongqing 400038, China; ${ }^{4}$ Section of Digestive Diseases, Yale University of Medicine, New Haven, CT, USA

Contributions: (I) Conception and design: Z Liang, X Ouyang; (II) Administrative support: Z Liang; (III) Provision of study materials or patients: H Pan, X Lei, L Chen; (IV) Collection and assembly of data: W Leng, H Pan, M Wu, X Lei, Q Wu; (V) Data analysis and interpretation: W Leng, M $\mathrm{Wu}$; (VI) Manuscript writing: All authors; (VII) Final approval of manuscript: All authors.

"These authors contributed equally to this work.

Correspondence to: Ziwen Liang. Department of Endocrinology, Southwest Hospital, the Third Military Medical University (Army Medical University), Chongqing 400038, China. Email: ziwenliang99@163.com.

Background: Diabetes mellitus (DM) is considered as a risk factor for the progress of liver diseases. After tissue damage, there is the highest amplitude of ubiquitously sterile inflammatory response in the liver, resulting in a major clinical consequence concerning a high prevalence of steatohepatitis in DM patients. This study aimed to investigate the inhibitory efficacy of dapagliflozin (DAPA), a sodium glucose cotransporter-2 (SGLT2) inhibitor, on experimental steatohepatitis with DM.

Methods: DM-steatohepatitis model was established by dual intraperitoneal injection of streptozotocin (STZ) and feeding with the high-fat diet (HFD) in apolipoprotein E-deficient $\left(\right.$ ApoE $\left.^{-/}\right)$mice $(\mathrm{n}=40)$. The mice were concurrently treated with DAPA $(1 \mathrm{mg} / \mathrm{kg} / \mathrm{d})$ by gavage for 12 weeks.

Results: In $\mathrm{ApoE}^{-/-}$mice, dual HFD/STZ dramatically induced hepatic damage and inflammation as compared with HFD alone. DAPA treatment was effective to protect from hepatic damage and inflammation in dual HFD/STZ treated $\mathrm{ApoE}^{-/-}$mice. DAPA also significantly the probability decreased the blood glucose, hepatic lipid accumulation, liver steatosis, and fibrotic response in dual HFD/STZ treated ApoE ${ }^{-/-}$ mice. Further mechanistic investigations indicated that the protection of DAPA on diabetic liver injury was associated with the suppressed production of hepatic reactive oxygen species (ROS) and malondialdehyde (MDA) and the inhibited activation of NOD-like receptor family pyrin domain containing 3 (NLRP3) inflammasome.

Conclusions: These data demonstrate the efficacy of DAPA for protecting liver damage, inflammation and steatosis from experimental steatohepatitis with DM, and indicate a possible involvement of the inhibited activity of ROS-NLRP3 inflammasome.

Keywords: Dapagliflozin (DAPA); steatohepatitis; reactive oxygen species (ROS); NOD-like receptor family pyrin domain containing 3 (NLRP3) inflammasome

Submitted Jun 22, 2019. Accepted for publication Aug 21, 2019.

doi: $10.21037 / \mathrm{atm} .2019 .09 .03$

View this article at: http://dx.doi.org/10.21037/atm.2019.09.03 


\section{Introduction}

Diabetes mellitus (DM) is one of the health priorities in the $21^{\text {st }}$ century, and a major global threat to the health of human beings. It was estimated that in 2017 there are 451 million (age 18-99 years) people with diabetes worldwide. These figures were expected to increase to 693 million by 2045 (1). Liver disease is one of the symptoms of DM. Patients with DM have an increased risk of developing nonalcoholic fatty liver disease (NAFLD), nonalcoholic steatohepatitis (NASH), hepatic fibrosis/ cirrhosis, and hepatocellular carcinoma (HCC) (2). The liver plays a vital role in the regulation of blood glucose and lipid metabolism and is a crucial organ of oxidative processes and free radical reactions. The biomarkers of oxidative stress like reactive oxygen species (ROS) are enhanced in liver tissues at the early stage of DM. The recent studies have shown that hyperglycemia and a pathological increase of free fatty acids (FFAs) are considered as the critical mediators of ROS production, which is known as triggering oxidative liver damage in $\operatorname{DM}(3,4)$.

The development of sterile inflammation after cell death is a ubiquitous response which occurs in all organs. However, the amplitude of sterile inflammatory response varies widely, and the liver is notable for developing an exceptionally strong sterile inflammatory response (5) which is seen in experimental models including the metabolic syndrome-associated development of NASH in patients with DM (6). The functional mechanism of sterile inflammation has been clarified in the studies of NOD-like receptor family pyrin domain containing 3 (NLRP3) inflammasome comprising NLRP3 protein, ASC adapter and pro-caspase-1. The ablation of NLRP3 inflammasome in mice has been shown to prevent obesityinduced inflammation in fat depots and liver as well as enhance insulin signaling (7). In addition, the previous reports have suggested that unlimited NLRP3 activation can lead to severe liver inflammation characterized by the infiltration of inflammatory cells and the activation of hepatic stellate cells (HSCs) with collagen deposition in the liver $(8,9)$. A large number of studies have shown that excessive ROS can result in the activation of NLRP3 inflammasome $(10,11)$. Due to the high metabolic activity of hepatocytes, the liver is a major organ attacked by ROS, which is responsible for not only deriving sterile inflammation, but also activating HSCs and extracellular matrix in the liver.

Sodium glucose cotransporter-2 (SGLT2) inhibitors demonstrate a unique antidiabetic mechanism of action related to the reduction of blood glucose by increasing urinary glucose excretion. We found that dapagliflozin (DAPA), a highly selective SGLT2 inhibitor, attenuated the formation of atherosclerotic lesions and the inflammation in diabetic atherosclerosis (12). Interestingly, we noted a difference in the appearance between the treated and untreated groups when dissecting these mice in the previous study (12), so we further studied the beneficial effects of DAPA on liver diseases.

Feeding the mice with a high-fat diet (HFD) resulted in the development of insulin resistance and DM (13). In addition, the mice injected with streptozotocin (STZ) developed damage to pancreas islet $\beta$ cells, with a decrease in insulin release (14). In accordance with the development of DM, an ideal animal model of DM was established by a combination of HFD and STZ injection, and it was used frequently to understand the pathogenesis and therapies of $\mathrm{DM}$ and its complications $(15,16)$. Hyperlipidemia-prone apolipoprotein E-deficient $\left(\mathrm{ApoE}^{--}\right)$mice were capable to develop hepatic steatosis and advanced fibrosis (17). Furthermore, the increasing evidences have demonstrated the presence of oxidative stress, necro-inflammatory changes, upregulation of pro-inflammatory genes, and macrophage infiltration in the livers of $\mathrm{ApoE}^{---}$mice fed with HFD (18). ApoE ${ }^{-/}$mice treated with HFD and STZ were generally companied with severe liver injury mainly in the form of inflammation and hepatocyte damage $(15,19)$. Thus, dual HFD-fed and STZ injection of $\mathrm{ApoE}^{-/-}$mice may provide an ideal animal model used for studying diabetic liver injury. Therefore, the animal model, established by dual treatment of HFD feeding and STZ injection to Apo: $\mathrm{E}^{-/-}$mice in our previous study about atherosclerotic lesions (12), is also applicable to studying liver injury, and to further investigating the effects and underling mechanism of DAPA on liver injury in DM and non-diabetes mellitus (NDM).

\section{Methods}

\section{Animals and treatment}

The animal experiments were performed in the Experimental Animal Platform of Medical Research Center in Army Medical University, and all animal research was approved by the Institutional Animal Ethics Committee/ Institutional Animal Care and Use Committee (IACUC) of Army Medical University. The methods, reported in the 
previous study (12), to establish the diabetic atherosclerotic mouse model is also applicable to establishing the diabetic liver injury model. Briefly, the $\mathrm{ApoE}^{-/-}$mice were fed with HFD plus STZ $(130 \mathrm{mg} / \mathrm{kg})$ injection, and the mice with blood glucose $\geq 16.7 \mathrm{mmol} / \mathrm{L}$ were chosen as the diabetic group. The experimental groups were divided into 5 groups as follows: (I) diabetic $\mathrm{ApoE}^{-/-}$mice receiving DAPA (DM + DAPA group, $\mathrm{n}=12)$; (II) diabetic $\mathrm{ApoE}^{-/-}$ mice receiving vehicle (DM + con group, $\mathrm{n}=8)$; (III) nondiabetic mice receiving DAPA (NDM + DAPA group, $\mathrm{n}=12)$; (IV) nondiabetic mice receiving vehicle (NDM + con group, n=8); and (V) wild-type (WT) C57BL/6J mice (WT control group, $n=6$ ). The mice were administrated with DAPA (AstraZeneca, USA) intragastrically $1.0 \mathrm{mg} / \mathrm{kg} / \mathrm{d}$ for 12 weeks, while $\mathrm{C} 57 \mathrm{BL} / 6 \mathrm{~J}$ and $\mathrm{ApoE}^{-1-}$ mice in the control group were administered with the vehicle.

\section{Biochemical measurement}

At an age of 28 weeks, all mice were subject to $8-12 \mathrm{~h}$ fasting and then anesthetized by intraperitoneal injection of $10 \%$ chloral hydrate. Thereafter, the blood was collected via orbital vein. The liver tissues preserved at $-80^{\circ} \mathrm{C}$ were weighed and cut into small pieces for homogenization. Then, they were centrifuged $\left(4^{\circ} \mathrm{C}, 4,000 \mathrm{rpm} \times 15 \mathrm{~min}\right)$ to separate the supernatant. Lipids in the supernatant were extracted, and the hepatic triglycerides (TGs) (Applygen, Beijing, China), FFAs (Jiancheng, Nanjing, China) concentrations were determined according to the manufacturer's instructions. The serum levels of total cholesterol (TCH), TG, high-density lipoprotein cholesterol (HDL-C), low-density lipoprotein cholesterol (LDL-C), FFAs, alanine aminotransferase (ALT), and aspartate aminotransferase (AST) were assessed by the Clinical Laboratory of Southwest Hospital, and those of IL-1 $\beta$ and IL-18 proteins were measured using enzymelinked immunosorbent assay (ELISA) kits (Shanghai Jonln Holding Co., Ltd., Shanghai, China) according to the manufacturer's instructions.

The malondialdehyde (MDA) contents and the total superoxide dismutase (SOD) activities in mouse livers were measured using an in vitro lipid peroxidation MDA assay kit (Nanjing Jiancheng Bioengineering Institute, Nanjing, China) and SOD analysis kit (Beyotime Institute of Biotechnology, Shanghai, China), respectively. Both measurements followed the manufacturer's instructions. Protein concentrations in samples were determined using a bicinchoninic acid (BCA) assay kit (Beyotime Institute of Biotechnology, Shanghai, China). MDA was expressed as $\mathrm{nmol} / \mathrm{mg}$ tissue protein while the activity of SOD was expressed as $\mathrm{U} / \mathrm{mg}$ tissue protein.

\section{Detection of ROS}

Freshly liver tissues were embedded in tissue-freezing compound and the specimens were cut into $5 \mu \mathrm{m}$ sections and placed on cover slips. To investigate the hepatic ROS levels, the liver tissues were incubated in the dark with $1 \mu \mathrm{mol} / \mathrm{L}$ dihydroethidium (DHE) (Beyotime Institute of Biotechnology, Shanghai, China) for 30 minutes at $37^{\circ} \mathrm{C}$. Next, the samples were washed 3 times in phosphatebuffered saline (PBS). To quantify DHE fluorescence, the glass slides were placed under confocal fluorescence microscope (Zeiss LSM 780), and DHE turns red fluorescent upon oxidation. The fluorescence intensities were quantified on Image-pro plus 6.0.

\section{Histological and immunofuorescence analyses}

Formalin-fixed liver tissues were made into $5 \mu \mathrm{m}$ thick paraffin sections, and then these sections were stained with hematoxylin and eosin (HE). For the evaluation of hepatic steatosis, the frozen liver sections were stained with Oil red O (Sigma, USA). The positive stained area was analyzed by Image-pro plus 6.0. To observe the degree of fibrosis and inflammation in the liver, the frozen sections were blocked at $4{ }^{\circ} \mathrm{C}$ overnight with primary antibodies ( $\alpha$-SMA antibody 1:400, Sigma; MOMA-2 antibody 1:50, Abcam) and then incubated with secondary antibodies for $2 \mathrm{~h}$. The resultant immunofluorescence was observed using a confocal fluorescence microscope and the positive staining was analyzed by quantifying the Indian Ocean Dipole (IOD) using Image pro plus 6.0.

\section{Western blot}

The protein samples were separated by sodium dodecyl sulfate-polyacrylamide gel electrophoresis (SDS-PAGE) and transferred onto a polyvinylidene difluoride (PVDF) membrane (Millipore Company, USA). The membrane was incubated overnight at $4{ }^{\circ} \mathrm{C}$ with primary antibodies (antiNLRP3 antibody 1:1,000; anti-caspase-1 antibody 1:400; anti-IL-1 $\beta$ antibody 1:1,000; and anti-IL-18 antibody 1:500; Novus Biologicals, Littleton, CO, USA) and 
A $\quad \mathrm{DM}+\mathrm{DAPA}$


$\mathrm{DM}+\mathrm{con}$


$N D M+D A P A$

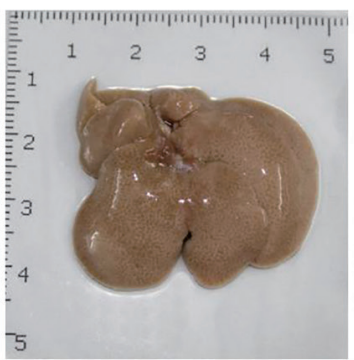

$\mathrm{NDM}+\mathrm{con}$



C57

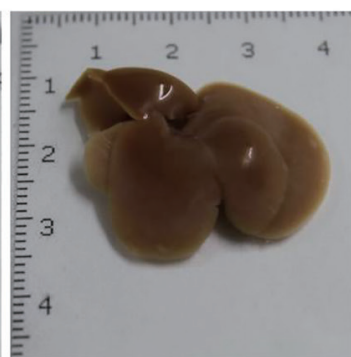

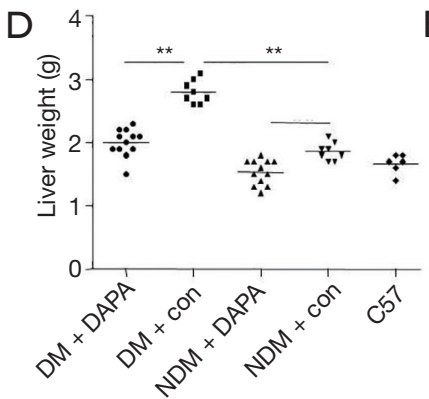

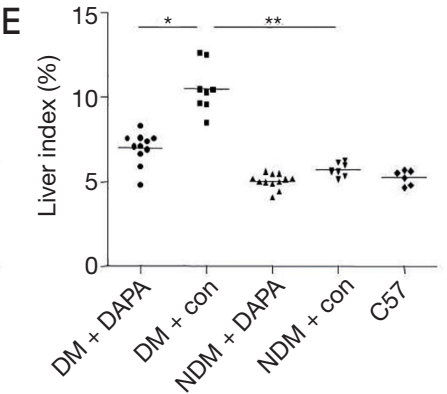

Figure 1 DAPA reduced liver damage in DM mice. (A) Representative photographs of gross examination of mouse livers; determination of serum ALT (B) and AST (C) levels; (D) effects of DAPA on the LW of mice; (E) effects of DAPA on the liver index of mice. The values are $\mathrm{M} \pm$ SEM. *, $\mathrm{P}<0.05$; **, $\mathrm{P}<0.01$. DAPA, dapagliflozin; DM, diabetes mellitus; LW, liver weight; ALT, alanine aminotransferase; AST, aspartate aminotransferase; $M$, mean; SEM, standard error of the mean; NDM, non-diabetes mellitus.

glyceraldehyde-3-phosphate dehydrogenase (GAPDH). It was then exposed to a horseradish peroxidase (HRP)labeled secondary antibody $(1: 5,000)$ and preserved at room temperature for two hours. The signals were detected with a chemiluminescent reagent specific for PVDF in a $1-3 \mathrm{~min}$ enhanced reaction (Thermo Fisher Scientific, USA). The levels of target proteins were normalized with GAPDH. The membrane was scanned with the molecular imager ChemiDoc XRS+ system (BIO-RAD, USA) and quantified using image lab software version 2.0.1. The protein expression level was detected three times for each sample.

\section{Statistical analysis}

The quantitative analysis was performed using SPSS Statistics 20.0. The data were expressed as the mean \pm standard deviation $(M \pm S D)$ at least repeat three times. The differences between groups were assessed using the non-parametric rank sum test for heterogeneity of variance or analysis of variance for homogeneity of variance; a difference with $\mathrm{P}<0.05$ was considered significant.

\section{Results}

\section{$D A P A$ affected the metabolic parameters and reduced liver injury and morphological damage in dual HFD/STZ treated ApoE ${ }^{-/}$mice}

Compared with control or non-STZ (NDM) treated groups, the elevated indicators of hepatic injury (e.g., serum transaminases, including AST and ALT) and the significant changes of liver morphology were seen after dual HFD/STZ treatment (DM) (Figure 1A,B,C). Despite no significant differences in body weight among the five groups, the liver weight (LW) and the liver to body weight ratio (LI) were increased in dual HFD/STZ treated group as compared with HFD group and WT control group (Figure 1D,E). DAPA treatment significantly reduced LW and LI in DM mice, and only decreased LW in NDM mice (Figure 1D,E). Interestingly, the treatment with DAPA throughout the experimental period caused the significant reduction of serum ALT and AST levels, the LW and LW/ body weight ratio, and improved liver morphology in dual HFD/STZ treated ApoE ${ }^{-/-}$mice (Figure 1). 
Table 1 Data on fasting blood glucose, and blood lipid profile in all groups

\begin{tabular}{lccccc}
\hline Parameters & DM + DAPA & DM + con & NDM + DAPA & NDM + con \\
\hline FBG (mmol/L) & $13.69 \pm 2.58^{\# \#}$ & $23.94 \pm 2.73$ & $9.17 \pm 2.28^{*}$ & $12.79 \pm 1.42$ & $12.05 \pm 2.24$ \\
TCH $(\mathrm{mmol} / \mathrm{L})$ & $34.34 \pm 7.97$ & $41.26 \pm 11.75$ & $29.56 \pm 5.28$ & $19.29 \pm 7.84$ & $0.48 \pm 0.41^{\& \&}$ \\
TG $(\mathrm{mmol} / \mathrm{L})$ & $0.87 \pm 0.22^{\# \#}$ & $2.14 \pm 1.15$ & $0.85 \pm 0.21$ & $0.78 \pm 0.19$ & $0.61 \pm 0.23$ \\
HDL-C (mmol/L) & $3.67 \pm 1.05$ & $3.50 \pm 1.73$ & $2.89 \pm 0.70$ & $2.12 \pm 0.74$ & $1.93 \pm 0.19$ \\
LDL-C (mmol/L) & $26.42 \pm 6.75$ & $25.71 \pm 8.85$ & $21.56 \pm 4.06$ & $18.43 \pm 3.06$ & $0.45 \pm 0.14^{\text {\&\& }}$ \\
FFA (mmol/L) & $1.02 \pm 0.22^{\#}$ & $1.31 \pm 0.34$ & $0.92 \pm 0.23$ & $0.83 \pm 0.24$ & $0.77 \pm 0.10$ \\
\hline
\end{tabular}

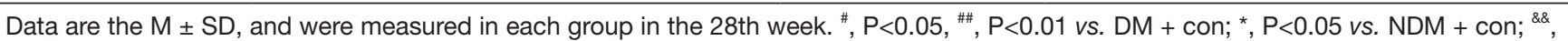
$\mathrm{P}<0.01$ vs. NDM + con. DM, diabetes mellitus; DAPA, dapagliflozin; NDM, non-diabetes mellitus; FBG, fasting blood glucose; TCH, total cholesterol; TG, triglyceride; HDL-C, high-density lipoprotein cholesterol; LDL-C, low-density lipoprotein cholesterol; FFA, free fatty acid; M, mean; SD, standard deviation.

Table 2 Scoring of hepatic steatosis in all groups

\begin{tabular}{lccccc}
\hline \multirow{2}{*}{ Group score } & \multicolumn{2}{c}{ Steatosis area [score] } & Score (M \pm SD) \\
\cline { 2 - 4 } & $<5 \%[0]$ & $5-33 \%[1]$ & $>33-66 \%[2]$ & $>66 \%[3]$ & 1 \\
DM + DAPA [12] & 0 & 2 & 9 & 4 & $1.83 \pm 0.58^{\star}$ \\
DM + con [8] & 0 & 0 & 4 & 0 & $0.37 \pm 0.74^{\# \#}$ \\
NDM + DAPA [12] & 5 & 5 & 2 & 0 & $0.75 \pm 0.75$ \\
NDM + con [8] & 2 & 4 & 0 & 0 & $1.00 \pm 0.76^{\&}$ \\
C57 [6] & 5 & 1 & 2 & $0.17 \pm 0.41$
\end{tabular}

Data are the $\mathrm{M} \pm \mathrm{SD}$. * $\mathrm{P}<0.05$, vs. $\mathrm{DM}+\mathrm{con} ;{ }^{\# \#}, \mathrm{P}<0.01$ vs. $\mathrm{NDM}+\mathrm{con} ;{ }^{\&}, \mathrm{P}<0.05$, vs. C57. DM, diabetes mellitus; DAPA, dapagliflozin; NDM, non-diabetes mellitus; $M$, mean; SD, standard deviation.

Our previous study (12) reported the effects of DAPA on blood glucose and blood lipid levels of mice (Table 1). The concentrations of blood glucose, TCH, TG, and FFA in DM mice were notably increased as compared with nonSTZ ApoE ${ }^{-1-}$ mice. After treatment with DAPA, the fasting blood glucose, TG and FFA levels were decreased in DM mice, while HDL-C, TCH, and LDL-C levels were merely affected.

\section{DAPA suppressed hepatic steatosis in dual HFD/STZ treated $\mathrm{ApoE}^{-1-}$ mice}

Compared with NDM mice, ApoE $E^{-/-}$mice under HFD feeding with STZ injection for a period of 12 weeks demonstrated several features of NASH, including hepatic damage, increased scores of macrovesicular steatosis (Table 2) and hepatic ballooning injury (Table 3) (Figure 2). Consistently, the quantification of Oil red O staining revealed that the lipid accumulation in hepatocytes
(Figure $3 A, B)$ and the contents of liver TG (Figure $3 C$ ) and FFAs (Figure $3 D$ ) were significantly higher in DM mice than in NDM mice. DAPA treatment significantly improved hepatic steatosis and NASH biochemical indicators in both DM and NDM mice (Figures 2,3). These results showed that DAPA reduced the degrees of hepatocellular damage, liver inflammation and lipid accumulation in $\mathrm{ApoE}^{-/-}$mice that were maintained by HFD in the diabetic condition.

\section{DAPA ameliorated liver fibrosis and inflammation}

The fibrotic response was measured in dual HFD/STZ treated $\mathrm{ApoE}^{-/-}$mice by $\alpha$-SMA immunofluorescent staining. The quantification of $\alpha$-SMA positive staining area revealed that dual HFD/STZ treatment significantly increased stellate cell activation and macrophage infiltration (Figure $4 A, B$ ). Such pro-fibrotic response was significantly attenuated by concurrent treatment 
Table 3 Scoring of hepatic ballooning injury in all groups

\begin{tabular}{lcccc}
\hline \multirow{2}{*}{ Group score } & \multicolumn{2}{c}{ Ballooning area [score] } & Score (M \pm SD) \\
\cline { 2 - 4 } DM + DAPA [12] & None [0] & Few balloon cells [1] & Many cells/prominent ballooning [2] & $1.25 \pm 0.45^{*}$ \\
DM + con [8] & 0 & 9 & 3 & $1.77 \pm 0.46^{\# \#}$ \\
NDM + DAPA [12] & 0 & 4 & 0 & $0.42 \pm 0.51$ \\
NDM + con [8] & 7 & 5 & 0 & $0.75 \pm 0.46^{\text {\&\& }}$ \\
C57 [6] & 2 & 6 & 0 & $0.00 \pm 0.00$ \\
\hline
\end{tabular}

Data are the $\mathrm{M} \pm \mathrm{SD}$. ${ }^{*}, \mathrm{P}<0.05$, vs. $\mathrm{DM}+\mathrm{con} ;{ }^{\# \#}, \mathrm{P}<0.01$ vs. $\mathrm{NDM}+\mathrm{con}^{\text {\&\& }}, \mathrm{P}<0.01$, vs. C57. DM, diabetes mellitus; DAPA, dapagliflozin; NDM, non-diabetes mellitus; M, mean; SD, standard deviation.

A
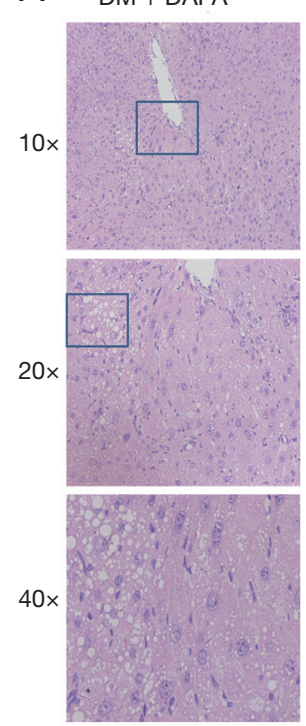

$\mathrm{DM}+\mathrm{con}$


$\mathrm{NDM}+\mathrm{DAPA}$
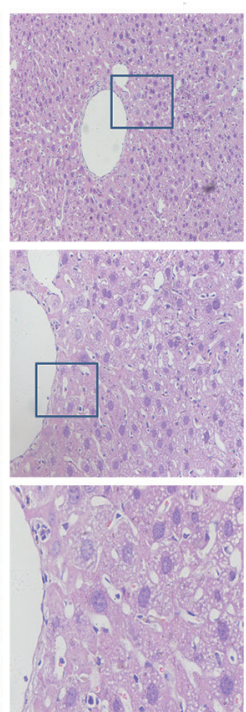

$\mathrm{NDM}+\mathrm{con}$
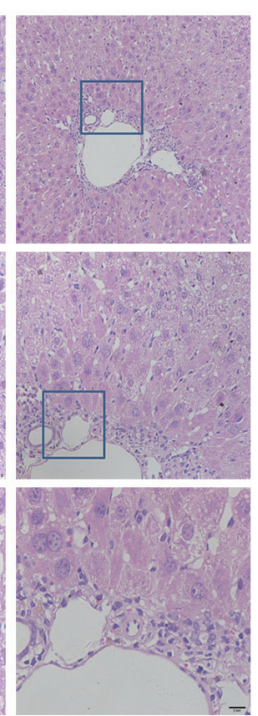

C57
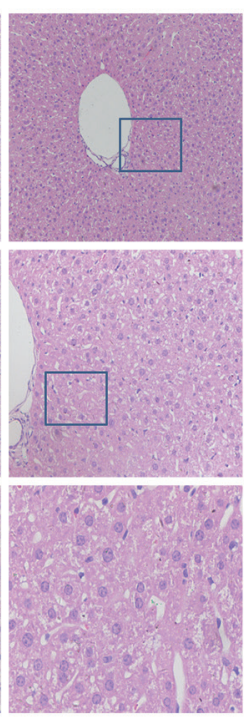

B

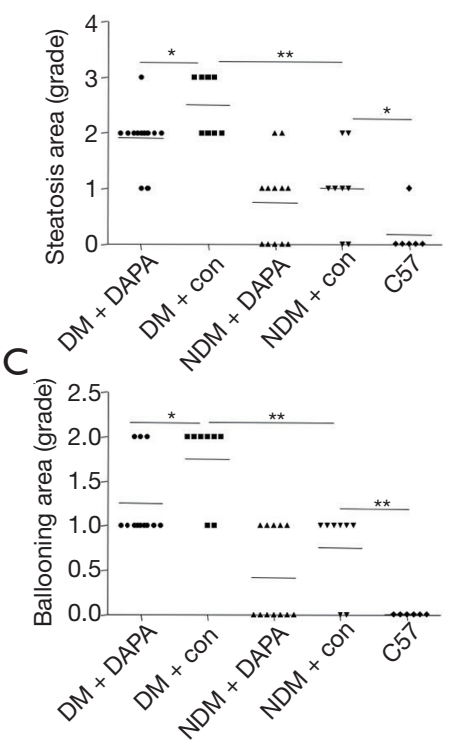

Figure 2 DAPA ameliorated hepatic steatosis in DM mice. (A) Liver sections were stained with HE; (B) scoring of hepatic steatosis in all groups; (C) scoring of hepatic ballooning injury in all groups. DAPA, dapagliflozin; DM, diabetes mellitus; HE, hematoxylin and eosin; NDM, non-diabetes mellitus.

with DAPA (Figure 4A,B). The liver immune cells (e.g., tissue-resident macrophages) were the key in controlling the inflammatory immune process and liver damage. As shown by immunofluorescence staining with MOMA-2 (a specific marker of monocyte/macrophages), the number of macrophages in the liver tissues was significantly increased in dual HFD/STZ treated ApoE ${ }^{-/}$mice (Figure 4B,C). DAPA showed remarkable protective effects against inflammatory cell infiltration in the liver under the DM condition (Figure 4B,C).

\section{DAPA attenuated oxidative stress associated with liver injury in dual HFD/STZ treated ApoE $E^{--}$mice}

We determined whether DAPA suppressed ROS production in the liver tissues from ApoE $\mathrm{E}^{-/}$. Consistently with the previous reports, diabetic liver injury caused the massive production of ROS in liver tissues in DM group compared with control group, as detected with DHE (a ROS fluorescent probe) (Figure 5A). Notably, DAPA significantly attenuated the production of ROS in DM and NDM mice (Figure 5B). The peroxidation of lipids contributed to the 
A

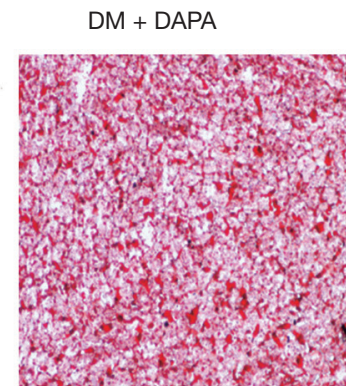

B

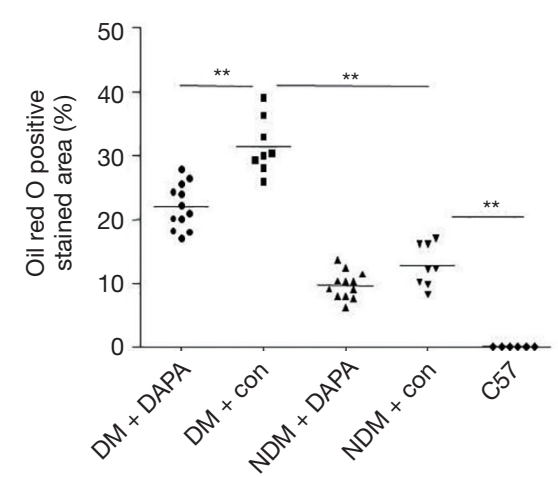

$\mathrm{DM}+\mathrm{con}$

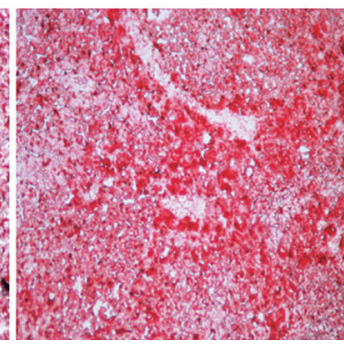

$\mathrm{NDM}+\mathrm{DAPA}$



$\mathrm{NDM}+\mathrm{con}$

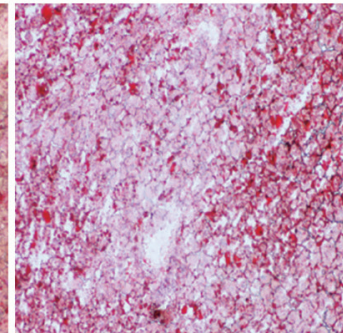

C57

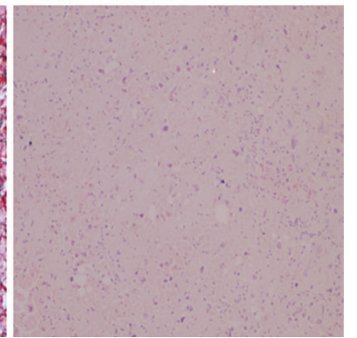

C

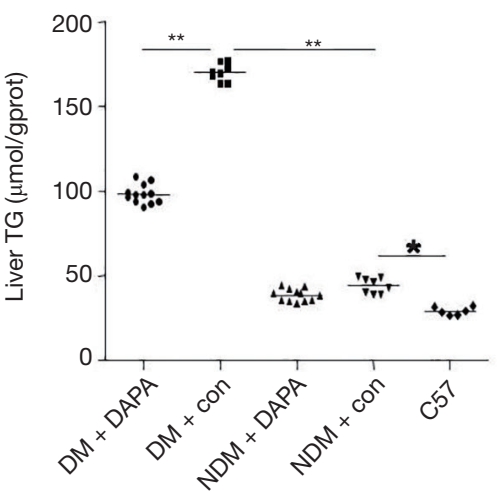

D



Figure 3 DAPA reduced the lipid content and pathological changes in the liver of DM mice. (A) Histological analysis of liver tissues by Oil red O staining; (B) the percentage of Oil red O positively stained area was analyzed by Image-pro plus 6.0; DAPA alleviated TG (C) and FFAs levels (D) in the liver of DM mice; . DAPA, dapagliflozin; DM, diabetes mellitus; NDM, non-diabetes mellitus; TG, hepatic triglyceride; FFA, free fatty acid.

production of MDA (a marker for lipid peroxidation). The MDA level in the liver was markedly greater in DM mice than in NDM mice, but lower in DM group and NDM group treated with DAPA (Figure 5C). Besides, the results also showed that SOD activity was increased in DAPAtreated DM group, but kept unchanged in NDM treatment group and control group (Figure 5D), indicating that DAPA reduced oxidation but caused no increase of antioxidation in NDM group.

\section{DAPA diminished the activity of bepatic NLRP3 inflammasome in dual HFD/STZ treated ApoE ${ }^{-/}$mice}

We further observed that the protein expression levels of NLRP3 inflammasome components, including NLRP3, caspase-1, IL-1 $\beta$ and IL-18, were significantly higher in NDM mice than in WT controls (Figure 6A) and lower in $\mathrm{NDM}_{\text {ApoE }}{ }^{-/}$mice than in DM mice. Western blot results showed that the protein expression levels of NLRP3 and caspase- 1 were decreased and the release of mature IL-1 $\beta$ and IL-18 was suppressed after DAPA treatment in DM mice. In addition, DAPA administration also reduced the expression of IL-1 $\beta$ in NDM ApoE ${ }^{-/}$mice (Figure $6 B$ ). Thus, these results indicated that DAPA attenuated the activation of NLRP3 inflammasome and then decreased the secretion of IL-1 $\beta$ and IL-18 in the liver of mice and especially DM mice. Besides, serum IL-1 $\beta$ and IL18 levels were increased in DM mice as compared with NDM ApoE $\mathrm{E}^{-/}$mice. DM mice demonstrated a marked increase in IL-1 $\beta$ and IL-18 levels which had a prominent reduction in control mice. The increasing trend of IL-1 $\beta$ and IL-18 levels were significantly diminished after DAPA administration in DM mice. In addition, the level of IL-1 $\beta$, but not IL-18, was also suppressed by DAPA in NDM mice (Figure 6C).

\section{Discussion}

Multiple studies have demonstrated that DM and NASH are closely related, DM can accelerate the progression of liver injury (2), and liver injury can aggravate abnormal glucose and lipid metabolism (20), thus increasing the 



B


Figure 4 DAPA reduced the hallmarks of fibrotic and macrophage infiltration in the liver of DM mice. (A) The liver sections were subjected to immunofluorescent staining by using $\alpha$-SMA antibody and DAPI for labeling activated stellate cells (red) and nuclei (blue) (200x); (B) the IOD of $\alpha$-SMA and MOMA-2 staining in livers of all groups was quantified; (C) using MOMA-2 antibody and DAPI for labeling tissue macrophages (red) and nuclei (blue) (200×). The values are $\mathrm{M} \pm$ SEM. * $\mathrm{P}<0.05 ;{ }^{* *}, \mathrm{P}<0.01$. DAPA, dapagliflozin; DM, diabetes mellitus; DAPI, 4',6-diamidino-2-phenylindole; IOD, Indian Ocean Dipole; M, mean; SEM, standard error of the mean; NDM, non-diabetes mellitus.

potential risks of cardiac-cerebral vascular and peripheral vascular events in DM patients (21). Therefore, it is vital to prevent liver injury during the management of DM. The studies have shown that SGLT2 inhibitors, a kind of novel oral hypoglycemic agents, can improve dyslipidemia, liver steatosis and NASH (22-24). Inflammasome activation was recently found as a key feature in mediating the sterile inflammatory response to a wide range of liver pathologies $(5,25)$. Our results showed that DAPA treatment effectively alleviated HFD/STZ-induced liver damage, inflammation and steatosis. DAPA effectively suppressed hepatic oxidative stress, i.e., the activity of NLRP3 inflammasome axis.

Hepatic steatosis was the first step in a spectrum of liver diseases termed NAFLD. The spectrum of disease ranged from simple steatosis to NASH, fibrosis and cirrhosis (26). ALT and AST are the hallmarks of both acute and chronic hepatic damages (27). Our results clearly presented that DAPA markedly reduced the serum levels of ALT and AST 
A

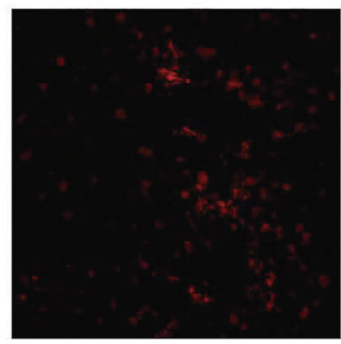

B

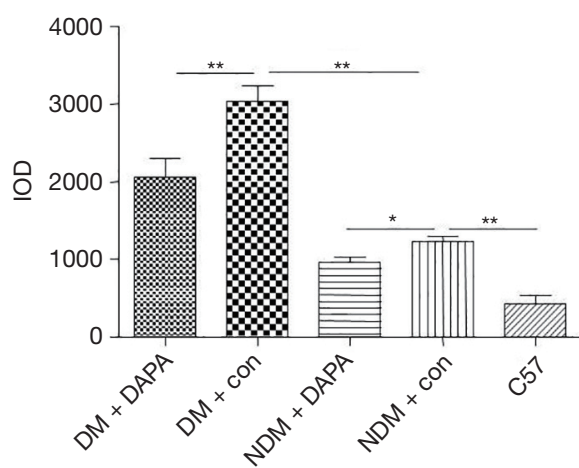

$\mathrm{DM}+\mathrm{con}$

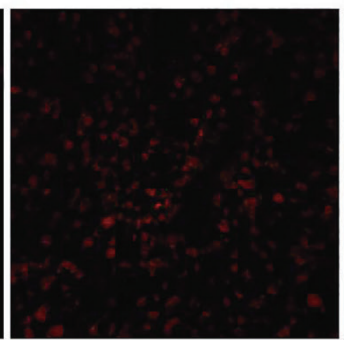

C
$\mathrm{NDM}+\mathrm{DAPA}$

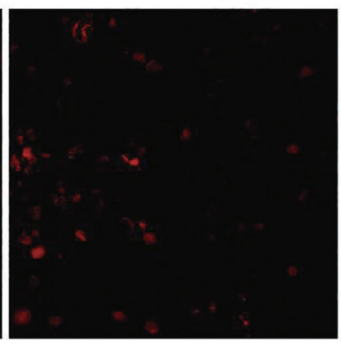

$\mathrm{NDM}+\mathrm{con}$

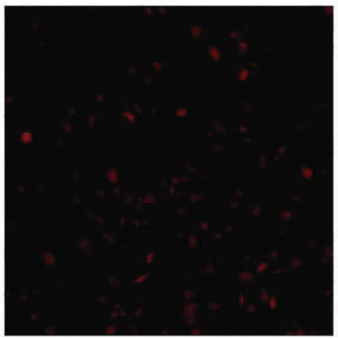

C57



D

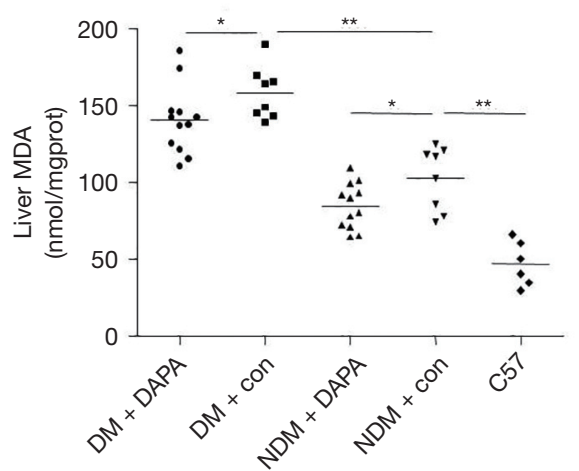

Figure 5 DAPA reduced ROS production in liver tissues of DM mice. (A) ROS production was determined by DHE staining; (B) the IOD of DHE staining in livers of all groups was quantified; (C) the lipid peroxidation product MDA was measured; (D) the activity of SOD in liver tissues of mice was tested. The values are $\mathrm{M} \pm \mathrm{SEM}$. * $\mathrm{P}<0.05 ;{ }^{*}, \mathrm{P}<0.01$. DAPA, dapagliflozin; ROS, reactive oxygen species; $\mathrm{DM}$, diabetes mellitus; DHE, dihydroethidium; IOD, Indian Ocean Dipole; MDA, malondialdehyde; SOD, superoxide dismutase; M, mean; SEM, standard error of the mean; NDM, non-diabetes mellitus.

and protected the liver from hepatic pathologic lesions in DM mice, suggesting that DAPA can suppress liver injury in DM. However, DAPA treatment had little effect on ALT and AST in NDM ApoE ${ }^{-/-}$mice, possibly because the liver injury of $\mathrm{NDM} \mathrm{ApoE}^{-/-}$mice did not reach the degree of hepatitis, so that DAPA exhibited minor effects. The pathogenesis of liver injury was considered as the involvement of multiple factors including lipotoxicity (28) usually caused by an increase in the flux of FFAs within hepatic cells. In the present study, we observed that DAPA reduced the serum and hepatic TG and FFAs levels, which corresponded to the reduced lipotoxicity in DM mice.

With the increase of time, steatosis and subsequently inflammation could lead to stellate cell activation and liver fibrosis. The presence and extent of fibrosis might be the most important factors in the prognosis of NAFLD and in predicting the risk of progression to cirrhosis and its complications (29). This study suggested that DAPA could improve liver fibrosis. According to our observations,
DAPA reduced the expression of IL-1 $\beta$ and IL-18 in the liver of DM mice, which promoted the recruitment of inflammatory cells to the liver and activated HSCs contributing to collagen deposition and liver fibrosis (30). Additionally, DAPA also decreased the hepatic infiltration of macrophages in these mice, which played a complex role in the progression and resolution of liver fibrosis (31-33). Therefore, we speculate that the improvement of liver fibrosis by DAPA treatment may be attributed to the reduced IL-1 $\beta$ production and macrophage infiltration in the liver.

Oxidative stress was defined as an elevated intracellular level of ROS that participated in the major course of inflammatory, metabolic and proliferative liver diseases (34). The importance of ROS in liver injury was shown by the observations that the reduction of ROS by a variety of manipulations protects the liver from a wide range of insults $(35,36)$. ROS plays a crucial role in the activation of NLRP3 inflammasome $(37,38)$. Suppressing 
A

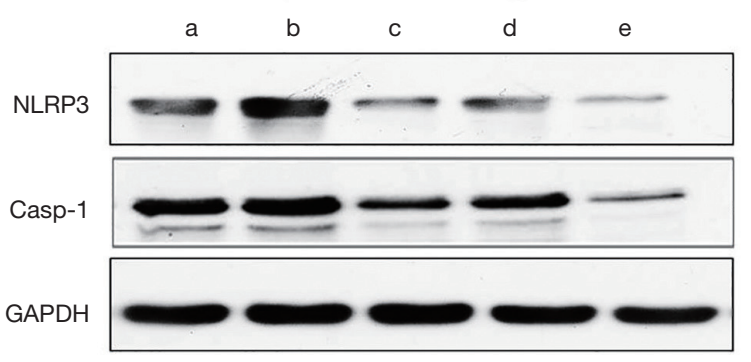

B



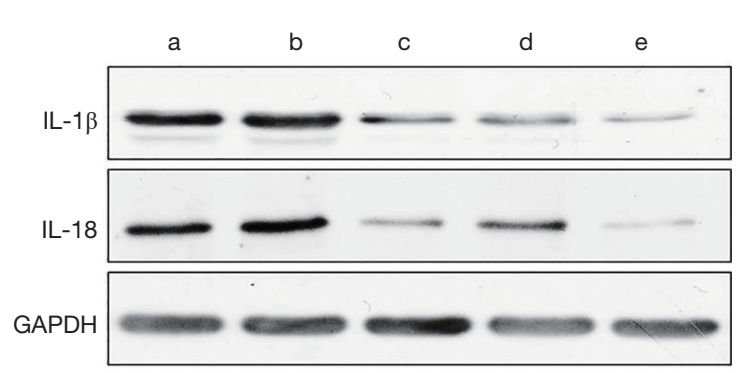

C



Figure 6 DAPA reduced the activity of NLRP3 inflammasome in the liver of DM mice. NLRP3, caspase-1, IL-1 $\beta$ and IL-18 were analyzed by Western blot. (A) GAPDH was used as a control with no changes in expression. a, DM + DAPA; b, DM + con; c, NDM + DAPA; d, NDM + con; e, C57; (B) the relative protein expression levels of NLRP3, caspase-1, IL-1 $\beta$ and IL-18 were quantified. a, DM + DAPA; b, DM + con; c, NDM + DAPA; d, NDM + con; e, C57; (C) the serum concentrations of IL-18 and IL-1 $\beta$ were measured in each group. The values are $M \pm S E M$. *, $\mathrm{P}<0.05$; **, $\mathrm{P}<0.01$. DAPA, dapagliflozin; NLRP3, NOD-like receptor family pyrin domain containing 3; DM, diabetes mellitus; GAPDH, glyceraldehyde-3-phosphate dehydrogenase; $M$, mean; SEM, standard error of the mean; NDM, non-diabetes mellitus.

the activation of NLRP3 inflammasome using a specific inhibitor can prevent HFD-induced liver damage $(39,40)$. In the present study, we firstly found that dual HFD and STZ-induced hepatic injury was sufficient to activate NLRP3 inflammasome in the liver. Similarly, DAPA decreased the serum IL-1 $\beta$ and IL-18 levels in DM mice, and also simultaneously reduced oxidative stress relevant to the decline of ROS and MDA and the increase of SOD. In addition, the inhibition of NLRP3 inflammasome by DAPA was associated with the attenuation of elevated blood glucose, FFAs and TG in DM mice fed with HFD, which is consistent with the previous studies. Thus, our study suggested that inflammasome activation induced by hyperglycemia and fatty acids was NLRP3-dependent and involved the elevated mitochondrial production of ROS.

Despite the protective effect of DAPA on the liver, the side effects of DAPA are not to be underestimated. Perry et al. recently confirmed DAPA can promote increased rates of hepatic ketogenesis and hepatic glucose production through a central mechanism resulting in stimulation of WAT lipolysis, this result provides a new mechanistic insight into the pathogenesis of SGLT2i-induced euglycemic ketoacidosis, alterations in hepatic glucose production and glucagon secretion in rodents (41).

In summary, this study has shown that DAPA treatment leads to the improvement of steatohepatitis associated with the inhibition of oxidative stress and the reduced activity of NLRP3 inflammasome in DM mice under experimental steatohepatitis. Furthermore, the administration of DAPA prevents the progression of liver steatosis, inflammation and fibrosis by inhibiting ROS and NLRP3 inflammasome activation. And DAPA may have clinically related potential hepatoprotective effects on steatohepatitis under the diabetic condition.

\section{Acknowledgments}

Funding: We thank AstraZeneca Plc. for providing 
dapagliflozin powder and this study was supported by grant from the National Natural Science Foundation of China (81670711).

\section{Footnote}

Conflicts of Interest: The authors have no conflicts of interest to declare.

Ethical Statement: The authors are accountable for all aspects of the work in ensuring that questions related to the accuracy or integrity of any part of the work are appropriately investigated and resolved. The animal experiments were performed in the Experimental Animal Platform of Medical Research Center in Army Medical University, and all animal research was approved by the Institutional Animal Ethics Committee/Institutional Animal Care and Use Committee (IACUC) of Army Medical University (No. AMUWEC2016121).

\section{References}

1. Cho NH, Shaw JE, Karuranga S, et al. IDF diabetes atlas: global estimates of diabetes prevalence for 2017 and projections for 2045. Diabetes Res Clin Pract 2018;138:271-81.

2. Armstrong MJ, Adams LA, Canbay A, et al. Extrahepatic complications of nonalcoholic fatty liver disease. Hepatology 2014;59:1174-97.

3. Yuan T, Yang T, Chen H, et al. New insights into oxidative stress and inflammation during diabetes mellitusaccelerated atherosclerosis. Redox Biol 2019;20:247-60.

4. Schmatz R, Perreira LB, Stefanello N, et al. Effects of resveratrol on biomarkers of oxidative stress and on the activity of delta aminolevulinic acid dehydratase in liver and kidney of streptozotocin-induced diabetic rats. Biochimie 2012;94:374-83.

5. Kubes P, Mehal WZ. Sterile inflammation in the liver. Gastroenterology 2012;143:1158-72.

6. Veena J, Muragundla A, Sidgiddi S, et al. Non-alcoholic fatty liver disease: need for a balanced nutritional source. Br J Nutr 2014;112:1858-72.

7. Rheinheimer J, de Souza BM, Cardoso NS, et al. Current role of the NLRP3 inflammasome on obesity and insulin resistance: a systematic review. Metabolism 2017;74:1-9.

8. Henao-Mejia J, Elinav E, Jin C, et al. Inflammasomemediated dysbiosis regulates progression of NAFLD and obesity. Nature 2012;482:179-85.
9. Ganz M, Csak T, Nath B, et al. Lipopolysaccharide induces and activates the Nalp3 inflammasome in the liver. World J Gastroenterol 2011;17:4772-8.

10. Hornung V, Bauernfeind F, Halle A, et al. Silica crystals and aluminum salts activate the NALP3 inflammasome through phagosomal destabilization. Nat Immunol 2008;9:847-56.

11. Halle A, Hornung V, Petzold GC, et al. The NALP3 inflammasome is involved in the innate immune response to amyloid-beta. Nat Immunol 2008;9:857-65.

12. Leng $W$, Ouyang X, Lei X, et al. The SGLT-2 inhibitor dapagliflozin has a therapeutic effect on atherosclerosis in diabetic ApoE-/- mice. Mediators Inflamm 2016;2016:6305735.

13. Heydemann A. An overview of murine high fat diet as a model for type 2 diabetes mellitus. J Diabetes Res 2016;2016:2902351.

14. Byun HR, Choi JA, Koh JY. The role of metallothionein-3 in streptozotocin-induced beta-islet cell death and diabetes in mice. Metallomics 2014;6:1748-57.

15. Wang K, Tang Z, Wang J, et al. Retracted: polysaccharide from Angelica sinensis ameliorates high-fat diet and STZinduced hepatic oxidative stress and inflammation in diabetic mice by activating the Sirt1-AMPK pathway. J Nutr Biochem 2017;43:88-97.

16. Subhasree N, Kamella A, Kaliappan I, et al. Antidiabetic and antihyperlipidemic activities of a novel polyherbal formulation in high fat diet/streptozotocin induced diabetic rat model. Indian J Pharmacol 2015;47:509-13.

17. Martínez-Clemente M, Ferré N, González-Périz A, et al. 5-lipoxygenase deficiency reduces hepatic inflammation and tumor necrosis factor alpha-induced hepatocyte damage in hyperlipidemia-prone ApoE-null mice. Hepatology 2010;51:817-27.

18. Sinha-Hikim I, Sinha-Hikim AP, Shen R, et al. A novel cystine based antioxidant attenuates oxidative stress and hepatic steatosis in diet-induced obese mice. Exp Mol Pathol 2011;91:419-28.

19. Nagaishi K, Ataka K, Echizen E, et al. Mesenchymal stem cell therapy ameliorates diabetic hepatocyte damage in mice by inhibiting infiltration of bone marrow-derived cells. Hepatology 2014;59:1816-29.

20. Ortiz-Lopez C, Lomonaco R, Orsak B, et al. Prevalence of prediabetes and diabetes and metabolic profile of patients with nonalcoholic fatty liver disease (NAFLD). Diabetes Care 2012;35:873-8.

21. Lizardi-Cervera J, Aguilar-Zapata D. Nonalcoholic fatty liver disease and its association with cardiovascular disease. 
Ann Hepatol 2009;8:S40-3.

22. Tahara A, Kurosaki E, Yokono M, et al. Effects of SGLT2 selective inhibitor ipragliflozin on hyperglycemia, hyperlipidemia, hepatic steatosis, oxidative stress, inflammation, and obesity in type 2 diabetic mice. Eur J Pharmacol 2013;715:246-55.

23. Honda Y, Imajo K, Kato T, et al. The selective SGLT2 inhibitor ipragliflozin has a therapeutic effect on nonalcoholic steatohepatitis in mice. PLoS One 2016;11:e0146337.

24. Qiang S, Nakatsu Y, Seno Y, et al. Treatment with the SGLT2 inhibitor luseogliflozin improves nonalcoholic steatohepatitis in a rodent model with diabetes mellitus. Diabetol Metab Syndr 2015;7:104.

25. Mehal WZ. The inflammasome in liver injury and nonalcoholic fatty liver disease. Dig Dis 2014;32:507-15.

26. Angulo P. Nonalcoholic fatty liver disease. N Engl J Med 2002;346:1221-31.

27. Zou Y, Li J, Lu C, et al. High-fat emulsion-induced rat model of nonalcoholic steatohepatitis. Life Sci 2006;79:1100-7.

28. Tilg H, Moschen AR. Evolution of inflammation in nonalcoholic fatty liver disease: the multiple parallel hits hypothesis. Hepatology 2010;52:1836-46.

29. Angulo P. Long-term mortality in nonalcoholic fatty liver disease: is liver histology of any prognostic significance? Hepatology 2010;51:373-5.

30. Wree A, Broderick L, Canbay A, et al. From NAFLD to NASH to cirrhosis-new insights into disease mechanisms. Nat Rev Gastroenterol Hepatol 2013;10:627-36.

31. Miura K, Kodama Y, Inokuchi S, et al. Toll-like receptor 9 promotes steatohepatitis by induction of interleukin-1beta in mice. Gastroenterology 2010;139:323-34.e7.

32. Ramachandran P, Pellicoro A, Vernon MA, et al. Differential Ly-6C expression identifies the recruited

Cite this article as: Leng $\mathrm{W}, \mathrm{Wu} M$, Pan $\mathrm{H}$, Lei $\mathrm{X}$, Chen $\mathrm{L}$, Wu Q, Ouyang X, Liang Z. The SGLT2 inhibitor Dapagliflozin attenuates the activity of ROS-NLRP3 inflammasome axis in steatohepatitis with diabetes mellitus. Ann Transl Med 2019;7(18):429. doi: 10.21037/atm.2019.09.03 macrophage phenotype, which orchestrates the regression of murine liver fibrosis. Proc Natl Acad Sci U S A 2012;109:E3186-95.

33. Baeck C, Wei X, Bartneck M, et al. Pharmacological inhibition of the chemokine C-C motif chemokine ligand 2 (monocyte chemoattractant protein 1 ) accelerates liver fibrosis regression by suppressing Ly-6C(+) macrophage infiltration in mice. Hepatology 2014;59:1060-72.

34. Cichoż-Lach H, Michalak A. Oxidative stress as a crucial factor in liver diseases. World J Gastroenterol 2014;20:8082-91.

35. Li S, Tan HY, Wang N, et al. The role of oxidative stress and antioxidants in liver diseases. Int J Mol Sci 2015;16:26087-124.

36. Cederbaum AI, Lu Y, Wu D. Role of oxidative stress in alcohol-induced liver injury. Arch Toxicol 2009;83:519-48.

37. Cruz CM, Rinna A, Forman HJ, et al. ATP activates a reactive oxygen species-dependent oxidative stress response and secretion of proinflammatory cytokines in macrophages. J Biol Chem 2007;282:2871-9.

38. Zhou R, Yazdi AS, Menu P, et al. A role for mitochondria in NLRP3 inflammasome activation. Nature 2011;469:221-5.

39. Wree A, Eguchi A, McGeough MD, et al. NLRP3 inflammasome activation results in hepatocyte pyroptosis, liver inflammation, and fibrosis in mice. Hepatology 2014;59:898-910.

40. Yang G, Lee HE, Lee JY. A pharmacological inhibitor of NLRP3 inflammasome prevents non-alcoholic fatty liver disease in a mouse model induced by high fat diet. Sci Rep 2016;6:24399.

41. Perry RJ, Rabin-Court A, Song JD, et al. Dehydration and insulinopenia are necessary and sufficient for euglycemic ketoacidosis in SGLT2 inhibitor-treated rats. Nat Commun 2019;10:548. 